\title{
Vaccine misadventure: inadvertent administration of tetanus toxoid
}

Background and epidemiology: A recent Morbidity and Mortality Weekly Report on inadvertent intradermal administration of tetanus toxoid ${ }^{1}$ highlights the importance of reporting adverse events that arise from vaccine administration. The US Vaccine Adverse Event Reporting System (VAERS) has detected 2 clusters of inadvertent intradermal administration of tetanus toxoid-containing vaccines in the past few months.

One cluster, reported in April 2004, involved 5 patients who received tetanus toxoid in error from a health care provider. A second cluster, which was detected 2 months later and involved an undisclosed number of patients, occurred when the health care provider confused tetanus and diphtheria toxoid (Td) with tuberculin purified protein derivative (PPD) and administered $\mathrm{Td}$ intradermally. The resulting local reactions $\therefore$ were interpreted as positive tuberculin skin tests, and the patients were given isoniazid. The error was detected when the vaccine lot numbers were reviewed and the product was revealed to be Td. The isoniazid therapy was discontinued, and no adverse reactions to it were observed.

As of March 2004, VAERS had reported about 100 events, spanning 21 states, of patients receiving tetanus toxoid-containing vaccines instead of PPD. This prompted the US Centers for Disease Control and Prevention and the Food and Drug Administration (FDA) to initiate a full review of adverse events caused by inadvertent administration of vaccines and PPD products reported to VAERS.

Clinical management: Similar packaging of PPD and tetanus toxoid-containing vaccines and side-by-side storage of these products in the refrigerator might contribute to medication error. Health care providers should consider the following measures to prevent vaccine confusion: physical separation of products in the refrigerator, careful visual inspection and reading of labels, preparation of PPD for patient use only at time of testing and improved record-keeping of lot numbers of vaccines and other injectable products.

Prevention: The FDA has recently introduced a rule requiring individual drug packages to have identifying bar codes. The goal is for health care facilities and pharmacies to scan packages when the drug is dispensed to avoid errors.

This report demonstrates the value in reporting errors ${ }^{2}$ in vaccine administration so that common mistakes can be identified and preventive measures introduced. In Canada, physicians and public health nurses could complete the form used to report vaccine-associated adverse events (www.hc-sc.gc.ca /pphb-dgspsp/dird-dimr/pdf/hc 4229e.pdf) and submit it to the local medical officer of health as well as to Health Canada's Division of Immunization.

\section{Erica Weir}

Associate Medical Officer of Health

Kingston, Frontenac and Lennox

\& Addington Health Unit

Kingston, Ont.

\section{References}

1. Notice to readers: Inadvertent intradermal administration of tetanus toxoidcontaining vaccines of tuberculosis skin tests. MMWR Morb Mortal Wkly Rep 2004;53(29):662-4. Available: www.cdc .gov/mmwr/preview/mmwrhtml/mm 5329a5.htm (accessed 2004 Aug 16).

2. Hébert P, Levin A, Robertson G. Bioethics for clinicians: 23. Disclosure of medical error. CMA7 2001; 164(4):509-13. 